\title{
Least-Squares-Based Iterative Identification Algorithm for Wiener Nonlinear Systems
}

\author{
Lincheng Zhou, ${ }^{1}$ Xiangli Li, ${ }^{1,2}$ and Feng Pan ${ }^{1}$ \\ ${ }^{1}$ Key Laboratory of Advanced Process Control for Light Industry (Ministry of Education), Jiangnan University, Wuxi 214122, China \\ ${ }^{2}$ Jiangsu College of Information Technology, Wuxi 214153, China \\ Correspondence should be addressed to Lincheng Zhou; zlcpk@163.com
}

Received 28 December 2012; Accepted 26 April 2013

Academic Editor: Hui-Shen Shen

Copyright (c) 2013 Lincheng Zhou et al. This is an open access article distributed under the Creative Commons Attribution License, which permits unrestricted use, distribution, and reproduction in any medium, provided the original work is properly cited.

\begin{abstract}
This paper focuses on the identification problem of Wiener nonlinear systems. The application of the key-term separation principle provides a simplified form of the estimated parameter model. To solve the identification problem of Wiener nonlinear systems with the unmeasurable variables in the information vector, the least-squares-based iterative algorithm is presented by replacing the unmeasurable variables in the information vector with their corresponding iterative estimates. The simulation results indicate that the proposed algorithm is effective.
\end{abstract}

\section{Introduction}

Wiener systems are typical nonlinear systems [1], which can represent a nonlinear dynamic system with a dynamic linear block followed by a nonlinear static function. Wiener systems have been used in modeling a glutamate fermentation process [2]. Recently, great attention has been paid to the identification issues for Wiener systems, and many studies have been performed. In much existing work, some assume that the nonlinear part of Wiener systems has an invertible function representation over the operating range of interest [3]. Wang and Ding presented least squares-based and gradient-based iterative identification algorithms for Wiener nonlinear systems [4]; Chen studied identification problems for Wiener systems with saturation and dead-zone nonlinearities [5]. Zhou et al. derived an auxiliary model-based gradient iterative algorithm for Wiener nonlinear output error systems by using the key-term decomposition principle [6].

The least-squares-based iterative algorithm is a class of basic identification algorithms in the field of system identification [7] and used in studying different types of systems, for example, multivariable systems [8-12] and multirate systems [13-16]. To estimate the parameters of systems in which the information vector contains unknown variables, the leastsquares-based iterative algorithms are usually used [17-22].
Ding et al. developed a least-squares-based iterative algorithm to estimate the parameters for a multi-input multioutput system with colored ARMA noise from input-output data [23]. On the basis of the work in [6, 24-26], this paper presents a least-squares-based iterative estimation algorithm for Wiener nonlinear systems.

The rest of this paper is organized as follows. Section 2 derives the identification model of Wiener nonlinear systems. Section 3 presents a least squares based iterative algorithm for Wiener nonlinear systems. Section 4 provides an example to illustrate the effectiveness of the proposed algorithm. The conclusions of the paper are summarized in Section 5.

\section{Problem Formation}

Let us firstly introduce some notations [27, 28]. The superscript T denotes the matrix transpose; I stands for an identity matrix of appropriate sizes; $\mathbf{1}_{n}$ represents an $n$-dimensional column vector whose elements are 1 ; the norm of a matrix $\mathbf{X}$ is defined by $\|\mathbf{X}\|^{2}:=\operatorname{tr}\left[\mathbf{X} \mathbf{X}^{\mathrm{T}}\right] ; \widehat{\mathbf{X}}(t)$ stands for the estimate of $\mathrm{X}$ at time $t$.

The Wiener nonlinear system consists of a linear dynamic subsystem followed by a static nonlinear block as shown in 


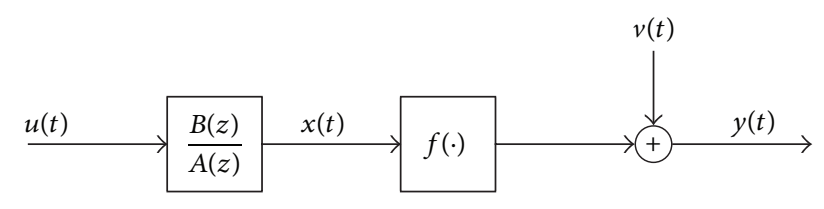

FIGURE 1: The Wiener nonlinear system.

Figure $1[25,26]$. The linear dynamic subsystem can be given as

$$
x(t)=\frac{B(z)}{A(z)} u(t),
$$

where $u(t)$ and $x(t)$ are the input and the inner output, respectively, and $A(z)$ and $B(z)$ are polynomials in $z^{-1}$, defined as

$$
\begin{aligned}
& A(z):=1+a_{1} z^{-1}+a_{2} z^{-2}+\cdots+a_{n_{a}} z^{-n_{a}}, \\
& B(z):=1+b_{1} z^{-1}+b_{2} z^{-2}+\cdots+b_{n_{b}} z^{-n_{b}} .
\end{aligned}
$$

The static nonlinear block is generally assumed to be the sum of the nonlinear basis functions of a known basis $\mathbf{f}:=\left(f_{1}\right.$, $f_{2}, \ldots, f_{n}$ ) as follows:

$$
\begin{aligned}
y(t) & =f(x(t)) \\
& =\gamma_{1} f_{1}(x(t))+\gamma_{2} f_{2}(x(t))+\cdots+\gamma_{n_{\gamma}} f_{n_{\gamma}}(x(t)) \\
& =\mathbf{f}(x(t)) \gamma .
\end{aligned}
$$

In this paper, we assume that the nonlinear function $f(\cdot)$ can be expressed by the polynomial of the order $n_{\gamma}$ as follows:

$$
f(x(t))=\gamma_{1} x(t)+\gamma_{2} x^{2}(t)+\cdots+\gamma_{n_{\gamma}} x^{n_{\gamma}}(t),
$$

and the polynomial orders $n_{\gamma}$ are known. Without loss of generality, we introduce a stochastic white noise $v(t)$ with zero mean and variance $\sigma^{2}$ to system output and have

$$
y(t)=f(x(t))+v(t) .
$$

The linear block output $x(t)$ is identical with the nonlinear block input. A direct substitution of $x(t)$ from (1) into (5) would result in a very complex expression containing crossmultiplied parameters and variables. To simplify this problem, the key-term separation principle can be applied [29]. We fix a coefficient of the nonlinear blocks. For example, let the first coefficient of $\gamma$ be unity; that is, $\gamma_{1}=1$. Equation (1) can be rewritten to be

$$
x(t)=[1-A(z)] x(t)+B(z) u(t),
$$

and then substituting (6) into (5) for the separated $x(t)$, the system output is given in the form

$$
y(t)=[1-A(z)] x(t)+B(z) u(t)+\sum_{i=2}^{n_{\gamma}} \gamma_{i} x^{i}(t)+v(t) .
$$

Define the information vectors and the parameter vectors

$$
\begin{aligned}
& \boldsymbol{\varphi}_{s}(t):=\left[-x(t-1),-x(t-2), \ldots,-x\left(t-n_{a}\right)\right. \text {, } \\
& \left.u(t-1), u(t-2), \ldots, u\left(t-n_{b}\right)\right]^{\mathrm{T}} \in \mathbb{R}^{n_{a}+n_{b}}, \\
& \boldsymbol{\varphi}_{\gamma}(t):=\left[x^{2}(t), x^{3}(t), \ldots, x^{n_{\gamma}}(t)\right]^{\mathrm{T}} \in \mathbb{R}^{n_{\gamma}-1}, \\
& \boldsymbol{\varphi}(t):=\left[\begin{array}{l}
\boldsymbol{\varphi}_{s}(t) \\
\boldsymbol{\varphi}_{\gamma}(t)
\end{array}\right] \in \mathbb{R}^{n_{0}}, \quad n_{0}=n_{a}+n_{b}+n_{\gamma}-1, \\
& \boldsymbol{\theta}_{s}:=\left[a_{1}, a_{2}, \ldots, a_{n_{a}}, b_{1}, b_{2}, \ldots, b_{n_{b}}\right]^{\mathrm{T}} \in \mathbb{R}^{n_{a}+n_{b}}, \\
& \boldsymbol{\theta}_{\gamma}:=\left[\gamma_{2}, \gamma_{3}, \ldots, \gamma_{n_{\gamma}}\right]^{\mathrm{T}} \in \mathbb{R}^{n_{\gamma}-1} \text {, } \\
& \boldsymbol{\theta}:=\left[\begin{array}{l}
\boldsymbol{\theta}_{s} \\
\boldsymbol{\theta}_{\gamma}
\end{array}\right] \in \mathbb{R}^{n_{0}} .
\end{aligned}
$$

Thus, (6) can be written in a vector form as

$$
x(t)=\boldsymbol{\varphi}_{s}^{\mathrm{T}}(t) \boldsymbol{\theta}_{s} .
$$

Using (9), from (7), we can obtain the following identification model:

$$
\begin{aligned}
y(t) & =\boldsymbol{\varphi}_{s}^{\mathrm{T}}(t) \boldsymbol{\theta}_{s}+\boldsymbol{\varphi}_{\gamma}^{\mathrm{T}}(t) \boldsymbol{\theta}_{\gamma}+v(t) \\
& =\boldsymbol{\varphi}^{\mathrm{T}}(t) \boldsymbol{\theta}+v(t) .
\end{aligned}
$$

The objective of this paper is to present a least squares based iterative algorithm to estimate the parameters $a_{i}, b_{i}, \gamma_{i}$ for the Wiener nonlinear system.

\section{The Least-Squares-Based Iterative Algorithm}

Based on the methods in $[18,20,24]$ for linear systems and Hammerstein nonlinear systems, we derive a least-squaresbased iterative algorithm for the Wiener model. Define the stacked output vector $\mathbf{Y}(L)$, the stacked information vector $\Phi(L)$, and the white noise vector $\mathbf{V}(L)$ as

$$
\begin{gathered}
\mathbf{Y}(L):=[y(L), y(L-1), \ldots, y(1)]^{\mathrm{T}} \in \mathbb{R}^{L}, \\
\boldsymbol{\Phi}(L):=[\boldsymbol{\varphi}(L), \boldsymbol{\varphi}(L-1), \ldots, \boldsymbol{\varphi}(1)]^{\mathrm{T}} \in \mathbb{R}^{L \times n_{0}}, \\
\mathbf{V}(L):=[v(L), v(L-1), \ldots, v(1)]^{\mathrm{T}} \in \mathbb{R}^{L} .
\end{gathered}
$$

Define a quadratic criterion function

$$
J(\boldsymbol{\theta}):=\|\mathbf{Y}(L)-\boldsymbol{\Phi}(L) \boldsymbol{\theta}\| .
$$

To minimize $J(\boldsymbol{\theta})$, letting its partial differential with respect to $\boldsymbol{\theta}$ be zero gives the least squares estimate of $\boldsymbol{\theta}$ as follows:

$$
\widehat{\boldsymbol{\theta}}=\left[\boldsymbol{\Phi}^{\mathrm{T}}(L) \boldsymbol{\Phi}(L)\right]^{-1} \boldsymbol{\Phi}^{\mathrm{T}}(L) \mathbf{Y}(L) .
$$

Since $\Phi(L)$ in (13) containing unknown inner variable $x(t)$ leads to a difficulty that the iterative solution $\widehat{\boldsymbol{\theta}}_{k}$ of $\boldsymbol{\theta}$ is 
impossible to compute. In order to solve this difficulty, the approach here is based on the auxiliary model idea [30-32]. Let $\hat{x}_{k}(t)$ be the estimate of $x(t)$ at iteration $k$ and $\widehat{\varphi}_{k}(t)$ the information vector $\varphi_{k}(t)$ obtained by replacing $x(t)$ with $\widehat{x}_{k}(t)$; that is,

$$
\widehat{\boldsymbol{\varphi}}_{k}(t)=\left[\begin{array}{l}
\widehat{\boldsymbol{\varphi}}_{s, k}(t) \\
\widehat{\boldsymbol{\varphi}}_{\gamma, k}(t)
\end{array}\right]
$$

with

$$
\begin{gathered}
\widehat{\boldsymbol{\varphi}}_{s, k}(t)=\left[-\widehat{x}_{k-1}(t-1),-\widehat{x}_{k-1}(t-2), \ldots,-\widehat{x}_{k-1}\left(t-n_{a}\right),\right. \\
\left.u(t-1), u(t-2), \ldots, u\left(t-n_{b}\right)\right]^{\mathrm{T}}, \\
\widehat{\boldsymbol{\varphi}}_{\gamma, k}(t)=\left[\hat{x}_{k-1}^{2}(t), \widehat{x}_{k-1}^{3}(t), \ldots, \hat{x}_{k-1}^{n_{\nu}}(t)\right]^{\mathrm{T}} .
\end{gathered}
$$

Replacing $\boldsymbol{\varphi}_{s}(t)$ and $\boldsymbol{\theta}_{s}$ in (9) with $\widehat{\boldsymbol{\varphi}}_{s, k}(t)$ and $\widehat{\boldsymbol{\theta}}_{s, k}$, respectively, the iterative estimate $\widehat{x}_{k}(t)$ can be obtained by the following auxiliary model:

$$
\widehat{x}_{k}(t)=\widehat{\boldsymbol{\varphi}}_{s, k}^{\mathrm{T}}(t) \widehat{\boldsymbol{\theta}}_{s, k}, \quad t=1,2, \ldots, L .
$$

Define

$$
\widehat{\boldsymbol{\Phi}}_{k}(L):=\left[\widehat{\boldsymbol{\varphi}}_{k}(L), \widehat{\boldsymbol{\varphi}}_{k}(L-1), \ldots, \widehat{\boldsymbol{\varphi}}_{k}(1)\right]^{\mathrm{T}} \in \mathbb{R}^{L \times n_{0}} .
$$

Let $\widehat{\boldsymbol{\theta}}_{k}=\left[\begin{array}{c}\hat{\boldsymbol{\theta}}_{s, k} \\ \widehat{\boldsymbol{\theta}}_{\gamma, k}\end{array}\right]$ be the estimate of $\boldsymbol{\theta}_{k}=\left[\begin{array}{l}\boldsymbol{\theta}_{s} \\ \boldsymbol{\theta}_{\gamma}\end{array}\right]$ at iteration $k$. Using $\widehat{\boldsymbol{\Phi}}_{k}(L)$ in place of $\boldsymbol{\Phi}(L)$ in (13), we have

$$
\widehat{\boldsymbol{\theta}}_{k}=\left[\widehat{\boldsymbol{\Phi}}_{k}^{\mathrm{T}}(L) \widehat{\boldsymbol{\Phi}}_{k}(L)\right]^{-1} \widehat{\boldsymbol{\Phi}}_{k}^{\mathrm{T}}(L) \mathbf{Y}(L) .
$$

Equations (13)-(18) form the least-squares-based iterative (LSI) identification algorithm for the Wiener nonlinear system, which can be summarized as [1]

$$
\begin{aligned}
& \widehat{\boldsymbol{\theta}}_{k}=\left[\widehat{\boldsymbol{\Phi}}_{k}^{\mathrm{T}}(L) \widehat{\boldsymbol{\Phi}}_{k}(L)\right]^{-1} \widehat{\boldsymbol{\Phi}}_{k}^{\mathrm{T}}(L) \mathbf{Y}(L), \\
& \widehat{\boldsymbol{\Phi}}_{k}(L)=\left[\widehat{\boldsymbol{\varphi}}_{k}(L), \widehat{\boldsymbol{\varphi}}_{k}(L-1), \ldots, \widehat{\boldsymbol{\varphi}}_{k}(1)\right]^{\mathrm{T}}, \\
& \mathbf{Y}(L)=[y(L), y(L-1), \ldots, y(1)]^{\mathrm{T}}, \\
& \widehat{\boldsymbol{\theta}}_{k}=\left[\widehat{\boldsymbol{\theta}}_{s, k}, \widehat{\boldsymbol{\theta}}_{\gamma, k}\right]^{\mathrm{T}}, \\
& \widehat{\boldsymbol{\varphi}}_{k}(t)=\left[\widehat{\boldsymbol{\varphi}}_{s, k}(t), \widehat{\boldsymbol{\varphi}}_{\gamma, k}(t)\right]^{\mathrm{T}}, \\
& \widehat{\boldsymbol{\varphi}}_{s, k}(t)=\left[-\widehat{x}_{k-1}(t-1),-\widehat{x}_{k-1}(t-2), \ldots,\right. \\
& \quad-\widehat{x}_{k-1}\left(t-n_{a}\right), u(t-1), \\
& \left.\quad u(t-2), \ldots, u\left(t-n_{b}\right)\right]^{\mathrm{T}}, \\
& \widehat{\boldsymbol{\varphi}}_{\gamma, k}(t)=\left[\widehat{x}_{k-1}^{2}(t), \widehat{x}_{k-1}^{3}(t), \ldots, \hat{x}_{k-1}^{n_{\gamma}}(t)\right]^{\mathrm{T}}, \\
& \widehat{x}_{k}(t)=\widehat{\boldsymbol{\varphi}}_{s, k}^{\mathrm{T}}(t) \widehat{\boldsymbol{\theta}}_{s, k}, \quad t=1,2, \ldots, L .
\end{aligned}
$$

The flowchart of computing the parameter estimate $\widehat{\boldsymbol{\theta}}_{k}$ is shown in Figure 2. The steps involved in computing the parameter estimate $\widehat{\boldsymbol{\theta}}_{k}$ in the LSI algorithm using a fixed data batch with the data length $L$ are listed as follows.

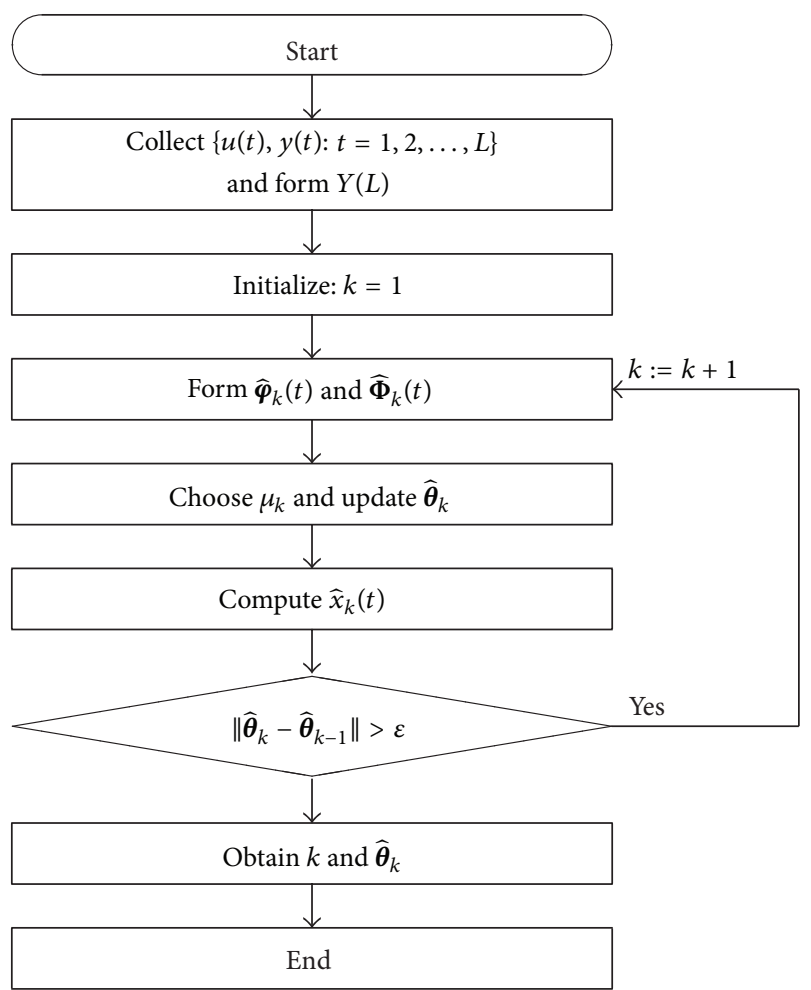

FIGURE 2: The flowchart of computing the LSI estimate $\widehat{\boldsymbol{\theta}}_{k}$.

(1) Collect the input-output data $\{u(t), y(t): t=0,1,2$, $\ldots, L\}$ and form $Y(L)$ by $(21)$.

(2) To initialize, let $k=1, \widehat{\boldsymbol{\theta}}_{0}=10^{-6} \mathbf{1}_{n_{0}}$, and $\widehat{x}_{0}(t)=10^{-6}$ for all $t$, and form $\widehat{\varphi}_{0}(t)$ by (23).

(3) Form $\widehat{\boldsymbol{\varphi}}_{k}(t)$ by (23) and $\widehat{\boldsymbol{\Phi}}_{k}(t)$ by (20).

(4) Update the estimate $\widehat{\boldsymbol{\theta}}_{k}$ by (19).

(5) Compute $\widehat{x}_{k}(t)$ by (24).

(6) For some preset small $\varepsilon$, if $\left\|\widehat{\boldsymbol{\theta}}_{k}-\widehat{\boldsymbol{\theta}}_{k-1}\right\|^{2} \leqslant \varepsilon$, then terminate the procedure and obtain the iterative times $k$ and estimate $\boldsymbol{\theta}_{k}$; otherwise, increment $k$ by 1 and go to step 3.

\section{Example}

Consider the following Wiener nonlinear system with the linear subsystem

$$
\begin{gathered}
x(t)=\frac{B(z)}{A(z)} u(t), \\
A(z)=1+a_{1} z^{-1}+a_{2} z^{-2}=1-0.18 z^{-1}+0.44 z^{-2}, \\
B(z)=1+b_{1} z^{-1}+b_{2} z^{-2}=1+0.58 z^{-1}+0.41 z^{-2},
\end{gathered}
$$

and the nonlinearity is given by

$$
\begin{aligned}
f(x(t)) & =\gamma_{1} x(t)+\gamma_{2} x^{2}(t)+\gamma_{3} x^{3}(t)+v(t) \\
& =x(t)-0.45 x^{2}(t)+0.25 x^{3}(t)+v(t) .
\end{aligned}
$$


TABLE 1: The parameter estimates $\left(a_{i}, b_{i}, \gamma_{i}\right)$ and their errors $\left(\sigma^{2}=0.10^{2}\right)$.

\begin{tabular}{lccccccc}
\hline$k$ & $a_{1}$ & $a_{2}$ & $b_{1}$ & $b_{2}$ & $\gamma_{2}$ & $\gamma_{3}$ \\
\hline 1 & 0.01390 & 0.05092 & 0.83611 & 0.74933 & -0.08844 & -0.00426 \\
2 & -0.25018 & 0.48270 & 0.60412 & 0.32843 & -0.23432 & 0.09158 \\
5 & -0.19276 & 0.43981 & 0.58302 & 0.40929 & -0.46012 & 0.25770 \\
10 & -0.16452 & 0.43173 & 0.58218 & 0.42255 & -0.45226 & 0.25192 & 29.18831 \\
20 & -0.17048 & 0.43263 & 0.58262 & 0.41964 & -0.45268 & 0.25202 & 2.37195 \\
30 & -0.17101 & 0.43273 & 0.58265 & 0.41937 & -0.45271 & 0.25203 \\
40 & -0.17106 & 0.43273 & 0.58265 & 0.41935 & -0.45271 & 0.25203 \\
50 & -0.17106 & 0.43273 & 0.58265 & 0.41935 & -0.45271 & 0.25203 \\
\hline True values & -0.18100 & 0.44000 & 0.58300 & 0.40800 & -0.45000 & 0.25000 \\
\hline
\end{tabular}

TABLE 2: The parameter estimates $\left(a_{i}, b_{i}, \gamma_{i}\right)$ and their errors $\left(\sigma^{2}=0.20^{2}\right)$.

\begin{tabular}{lccccccc}
\hline$k$ & $a_{1}$ & $a_{2}$ & $b_{1}$ & $b_{2}$ & $\gamma_{2}$ & $\delta(\%)$ \\
\hline 1 & 0.01254 & 0.05414 & 0.83538 & 0.75541 & -0.08800 & -0.00425 & 75.34758 \\
2 & -0.23806 & 0.47597 & 0.60817 & 0.34779 & -0.23179 & 0.08885 & 28.73714 \\
5 & -0.17964 & 0.43318 & 0.58596 & 0.42400 & -0.45097 & 0.24847 \\
10 & -0.15914 & 0.42794 & 0.58447 & 0.43252 & -0.44475 & 0.24437 & 3.78072 \\
20 & -0.16374 & 0.42887 & 0.58476 & 0.43020 & -0.44497 & 0.24442 & 3.12454 \\
30 & -0.16407 & 0.42894 & 0.58478 & 0.43003 & -0.44499 & 0.24443 \\
40 & -0.16410 & 0.42895 & 0.58478 & 0.43001 & -0.44499 & 0.24443 \\
50 & -0.16410 & 0.42895 & 0.58478 & 0.43001 & -0.44499 & 0.24443 \\
\hline True values & -0.18100 & 0.44000 & 0.58300 & 0.40800 & -0.45000 & 0.25000 \\
\hline
\end{tabular}

For this example system, $\{u(t)\}$ are taken as persistent excitation signal sequences with zero mean and unit variance and $\{v(t)\}$ as a white noise process with zero mean and constant variances $\sigma^{2}=0.10^{2}$ and $\sigma^{2}=0.20^{2}$, separately. Taking the data length $L=1000$, we apply the proposed LSI algorithm in (19)-(24) to estimate the parameters $\left(a_{i}, b_{i}, \gamma_{i}\right)$ of this system the parameter estimates and their errors with different noise variances are shown in Tables 1 and 2 , and the parameter estimation errors $\delta$ versus $k$ are shown in Figure 3, where $\delta:=$ $\left\|\widehat{\boldsymbol{\theta}}_{k}(t)-\boldsymbol{\theta}\right\| /\|\boldsymbol{\theta}\|$.

From Tables 1 and 2 and Figure 3, we can draw the following conclusions.

(i) The parameter estimation errors given by the proposed approach gradually become smaller as the iteration $k$ increases; see the error curves of the algorithm in Figure 3 and the estimation errors of the last columns of Tables 1 and 2.

(ii) As the variance of the noise decreases, the parameter estimation errors given by the proposed approach become smaller; see Tables 1 and 2.

\section{Conclusions}

In this paper, we have presented a least-squares-based iterative identification algorithm for Wiener nonlinear systems. Using the key-term separation principle, we construct the identification model of Wiener nonlinear systems. The proposed algorithm can directly estimate the parameters of the

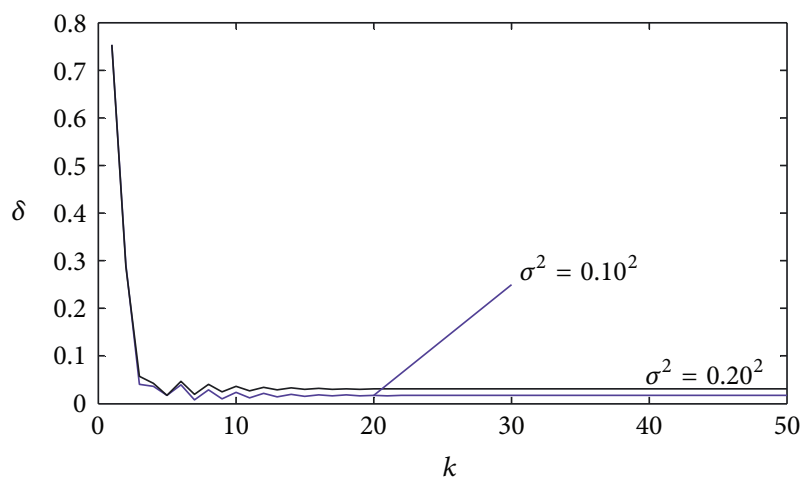

FIGURE 3: The estimation errors $\delta$ versus $k$.

linear subsystem and nonlinear part of Wiener nonlinear systems. The simulation results verified the effectiveness of the proposed algorithm. The proposed method for Wiener nonlinear systems can combine the auxiliary model identification idea [33-37], the multi-innovation identification theory [38-44], the bias compensation methods [45-48], and the maximum likelihood principle [49-52] to study identification problems of linear or nonlinear systems with colored noise and Hammerstein nonlinear systems [53-59].

\section{Acknowledgments}

This work was supported by the National Natural Science Foundation of China (no. 61273131), the University Graduate 
Scientific Research Innovation Program of Jiangsu Province (CXLX12_0722), the Fundamental Research Funds for the Central Universities (JUDCF11042 and JUDCF12031), the 111 Project (B12018), and PAPD of Jiangsu Higher Education Institutions.

\section{References}

[1] F. Ding, System Identification-New Theory and Methods, Science Press, Beijing, China, 2013.

[2] L. C. Zhou, X. L. Li, and F. Pan, "Gradient-based iterative identification for MISO Wiener nonlinear systems: application to a glutamate fermentation process," Applied Mathematics Letters, 2013.

[3] D. Q. Wang and F. Ding, "Hierarchical least squares estimation algorithm for Hammerstein-Wiener systems," IEEE Signal Processing Letters, vol. 19, no. 12, pp. 825-828, 2012.

[4] D. Q. Wang and F. Ding, "Least squares based and gradient based iterative identification for Wiener nonlinear systems," Signal Processing, vol. 91, no. 5, pp. 1182-1189, 2011.

[5] J. Chen, "Gradient-based iterative algorithm for Wiener systems with saturation and dead-zone nonlinearities," Journal of Vibration and Control, 2012.

[6] L. C. Zhou, X. L. Li, and F. Pan, "Gradient based iterative parameter identification for Wiener nonlinear systems," Applied Mathematical Modelling, vol. 37, 2013.

[7] D. Q. Wang, "Least squares-based recursive and iterative estimation for output error moving average systems using data filtering," IET Control Theory \& Applications, vol. 5, no. 14, pp. 1648-1657, 2011.

[8] Y. Liu, Y. Xiao, and X. Zhao, "Multi-innovation stochastic gradient algorithm for multiple-input single-output systems using the auxiliary model," Applied Mathematics and Computation, vol. 215, no. 4, pp. 1477-1483, 2009.

[9] Y. Liu, J. Sheng, and R. Ding, "Convergence of stochastic gradient estimation algorithm for multivariable ARX-like systems," Computers \& Mathematics with Applications, vol. 59, no. 8, pp. 2615-2627, 2010.

[10] H. Q. Han, L. Xie, F. Ding, and X. Liub, "Hierarchical least squares based iterative identification for multivariable systems with moving average noises," Mathematical and Computer Modelling, vol. 51, no. 9-10, pp. 1213-1220, 2010.

[11] Z. Zhang, F. Ding, and X. Liu, "Hierarchical gradient based iterative parameter estimation algorithm for multivariable output error moving average systems," Computers \& Mathematics with Applications, vol. 61, no. 3, pp. 672-682, 2011.

[12] Y. Liu and F. Ding, "Convergence properties of the least squares estimation algorithm for multivariable systems," Applied Mathematical Modelling, vol. 37, no. 1-2, pp. 476-483, 2013.

[13] F. Ding, L. Qiu, and T. Chen, "Reconstruction of continuoustime systems from their non-uniformly sampled discrete-time systems," Automatica, vol. 45, no. 2, pp. 324-332, 2009.

[14] J. Ding, F. Ding, X. P. Liu, and G. Liu, "Hierarchical least squares identification for linear SISO systems with dual-rate sampleddata," IEEE Transactions on Automatic Control, vol. 56, no. 11, pp. 2677-2683, 2011.

[15] X. Liu and J. Lu, "Least squares based iterative identification for a class of multirate systems," Automatica, vol. 46, no. 3, pp. 549$554,2010$.

[16] Y. Liu, F. Ding, and Y. Shi, "Least squares estimation for a class of non-uniformly sampled systems based on the hierarchical identification principle," Circuits, Systems, and Signal Processing, vol. 31, no. 6, pp. 1985-2000, 2012.

[17] Y. J. Liu, D. Q. Wang, and F. Ding, "Least-squares based iterative algorithms for identifying Box-Jenkins models with finite measurement data," Digital Signal Processing, vol. 20, no. 5, pp. 1458-1467, 2010.

[18] F. Ding, P. X. Liu, and G. Liu, "Identification methods for Hammerstein nonlinear systems," Digital Signal Processing, vol. 21, no. 2, pp. 215-238, 2011.

[19] F. Ding, "Decomposition based fast least squares algorithm for output error systems," Signal Processing, vol. 93, no. 5, pp. 12351242, 2013.

[20] F. Ding, X. G. Liu, and J. Chu, "Gradient-based and leastsquares-based iterative algorithms for Hammerstein systems using the hierarchical identification principle," IET Control Theory and Applications, vol. 7, 2013.

[21] D. Wang, R. Ding, and X. Dong, "Iterative parameter estimation for a class of multivariable systems based on the hierarchical identification principle and the gradient search," Circuits, Systems, and Signal Processing, vol. 31, no. 6, pp. 2167-2177, 2012.

[22] F. Ding, "Two-stage least squares based iterative estimation algorithm for CARARMA system modeling," Applied Mathematical Modelling, vol. 37, no. 7, pp. 4798-4808, 2013.

[23] F. Ding, Y. J. Liu, and B. Bao, "Gradient based and least squares based iterative estimation algorithms for multi-input multioutput systems," Proceedings of the Institution of Mechanical Engineers, Part I: Journal of Systems and Control Engineering, vol. 226, no. 1, pp. 43-55, 2012.

[24] F. Ding, P. X. Liu, and G. Liu, "Gradient based and leastsquares based iterative identification methods for $\mathrm{OE}$ and OEMA systems," Digital Signal Processing, vol. 20, no. 3, pp. 664-677, 2010.

[25] W. L. Xiong, J. X. Ma, and R. F. Ding, "An iterative numerical algorithm for modeling a class of Wiener nonlinear systems," Applied Mathematics Letters, vol. 26, no. 4, pp. 487-493, 2013.

[26] M. M. Liu, Y. S. Xiao, and R. F. Ding, "Iterative identification algorithm for Wiener nonlinear systems using the Newton method," Applied Mathematical Modelling, vol. 37, no. 9, pp. 6584-6591, 2013.

[27] F. Ding, G. Liu, and X. P. Liu, "Partially coupled stochastic gradient identification methods for non-uniformly sampled systems," IEEE Transactions on Automatic Control, vol. 55, no. 8, pp. 1976-1981, 2010.

[28] F. Ding, G. Liu, and X. P. Liu, "Parameter estimation with scarce measurements," Automatica, vol. 47, no. 8, pp. 1646-1655, 2011.

[29] J. Vörös, "Identification of nonlinear cascade systems with timevarying backlash," Journal of Electrical Engineering, vol. 62, no. 2, pp. 87-92, 2011.

[30] F. Ding, Y. Shi, and T. Chen, "Auxiliary model-based leastsquares identification methods for Hammerstein output-error systems," Systems \& Control Letters, vol. 56, no. 5, pp. 373-380, 2007.

[31] F. Ding and T. Chen, "Identification of Hammerstein nonlinear ARMAX systems," Automatica, vol. 41, no. 9, pp. 1479-1489, 2005.

[32] F. Ding, Y. Shi, and T. Chen, "Gradient-based identification methods for Hammerstein nonlinear ARMAX models," Nonlinear Dynamics, vol. 45, no. 1-2, pp. 31-43, 2006.

[33] F. Ding and T. Chen, "Combined parameter and output estimation of dual-rate systems using an auxiliary model," Automatica, vol. 40, no. 10, pp. 1739-1748, 2004. 
[34] F. Ding and T. Chen, "Identification of dual-rate systems based on finite impulse response models," International Journal of Adaptive Control and Signal Processing, vol. 18, no. 7, pp. 589598, 2004.

[35] F. Ding and J. Ding, "Least-squares parameter estimation for systems with irregularly missing data," International Journal of Adaptive Control and Signal Processing, vol. 24, no. 7, pp. 540553, 2010.

[36] F. Ding and T. Chen, "Parameter estimation of dual-rate stochastic systems by using an output error method," IEEE Transactions on Automatic Control, vol. 50, no. 9, pp. 1436-1441, 2005.

[37] F. Ding, "Hierarchical multi-innovation stochastic gradient algorithm for Hammerstein nonlinear system modeling," Applied Mathematical Modelling, vol. 37, no. 4, pp. 1694-1704, 2013.

[38] F. Ding and T. Chen, "Performance analysis of multi-innovation gradient type identification methods," Automatica, vol. 43, no. 1, pp. 1-14, 2007.

[39] F. Ding, X. P. Liu, and G. Liu, "Auxiliary model based multiinnovation extended stochastic gradient parameter estimation with colored measurement noises," Signal Processing, vol. 89, no. 10, pp. 1883-1890, 2009.

[40] F. Ding, "Several multi-innovation identification methods," Digital Signal Processing, vol. 20, no. 4, pp. 1027-1039, 2010.

[41] Y. Liu, L. Yu, and F. Ding, "Multi-innovation extended stochastic gradient algorithm and its performance analysis," Circuits, Systems, and Signal Processing, vol. 29, no. 4, pp. 649-667, 2010.

[42] D. Q. Wang and F. Ding, "Performance analysis of the auxiliary models based multi-innovation stochastic gradient estimation algorithm for output error systems," Digital Signal Processing, vol. 20, no. 3, pp. 750-762, 2010.

[43] F. Ding, X. P. Liu, and G. Liu, "Multi-innovation least squares identification for linear and pseudo-linear regression models," IEEE Transactions on Systems, Man, and Cybernetics B, vol. 40, no. 3, pp. 767-778, 2010.

[44] L. Xie, Y. J. Liu, H. Z. Yang, and F. Ding, "Modelling and identification for non-uniformly periodically sampled-data systems," IET Control Theory \& Applications, vol. 4, no. 5, pp. 784-794, 2010.

[45] Y. Zhang and G. Cui, "Bias compensation methods for stochastic systems with colored noise," Applied Mathematical Modelling, vol. 35, no. 4, pp. 1709-1716, 2011.

[46] Y. Zhang, "Unbiased identification of a class of multi-input single-output systems with correlated disturbances using bias compensation methods," Mathematical and Computer Modelling, vol. 53, no. 9-10, pp. 1810-1819, 2011.

[47] F. Ding, T. Chen, and L. Qiu, "Bias compensation based recursive least squares identification algorithm for MISO systems," IEEE Transactions on Circuits and Systems II: Express Briefs, vol. 53, no. 5, pp. 349-353, 2006.

[48] J. Ding and F. Ding, "Bias compensation-based parameter estimation for output error moving average systems," International Journal of Adaptive Control and Signal Processing, vol. 25, no. 12, pp. 1100-1111, 2011.

[49] W. Wang, F. Ding, and J. Dai, "Maximum likelihood least squares identification for systems with autoregressive moving average noise," Applied Mathematical Modelling, vol. 36, no. 5, pp. 1842-1853, 2012.

[50] J. Li and F. Ding, "Maximum likelihood stochastic gradient estimation for Hammerstein systems with colored noise based on the key term separation technique," Computers \& Mathematics with Applications, vol. 62, no. 11, pp. 4170-4177, 2011.
[51] J. Li, F. Ding, and G. Yang, "Maximum likelihood least squares identification method for input nonlinear finite impulse response moving average systems," Mathematical and Computer Modelling, vol. 55, no. 3-4, pp. 442-450, 2012.

[52] J. Li, "Parameter estimation for Hammerstein CARARMA systems based on the Newton iteration," Applied Mathematics Letters of Rapid Publication, vol. 26, no. 1, pp. 91-96, 2013.

[53] J. Li, R. Ding, and Y. Yang, "Iterative parameter identification methods for nonlinear functions," Applied Mathematical Modelling, vol. 36, no. 6, pp. 2739-2750, 2012.

[54] F. Ding and T. Chen, "Hierarchical identification of lifted statespace models for general dual-rate systems," IEEE Transactions on Circuits and Systems. I. Regular Papers, vol. 52, no. 6, pp. 11791187, 2005.

[55] F. Ding and T. Chen, "Hierarchical least squares identification methods for multivariable systems," IEEE Transactions on Automatic Control, vol. 50, no. 3, pp. 397-402, 2005.

[56] W. Xiong, W. Fan, and R. Ding, "Least-squares parameter estimation algorithm for a class of input nonlinear systems," Journal of Applied Mathematics, vol. 2012, Article ID 684074, 14 pages, 2012.

[57] X. L. Li, R. F. Ding, and L. C. Zhou, "Least squares based iterative identification algorithm for Hammerstein nonlinear systems with non-uniform sampling," International Journal of Computer Mathematics, 2013.

[58] P. P. Hu and F. Ding, "Multistage least squares based iterative estimation for feedback nonlinear systems with moving average noises using the hierarchical identification principle," Nonlinear Dynamics, vol. 52, 2013.

[59] F. Ding, "Coupled-least-squares identification for multivariable systems," IET Control Theory and Applications, vol. 7, no. 1, pp. 68-79, 2013. 


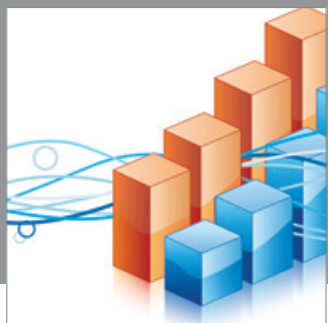

Advances in

Operations Research

mansans

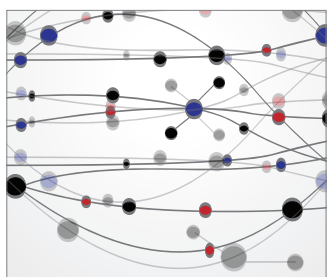

The Scientific World Journal
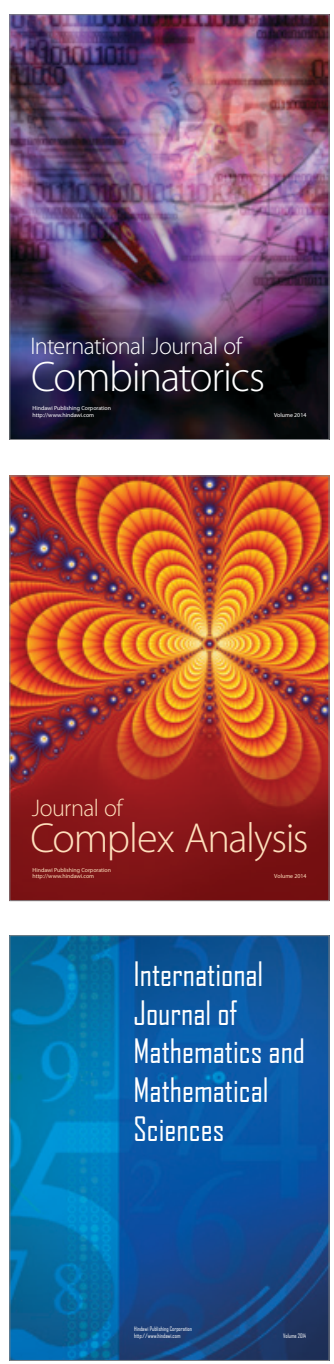
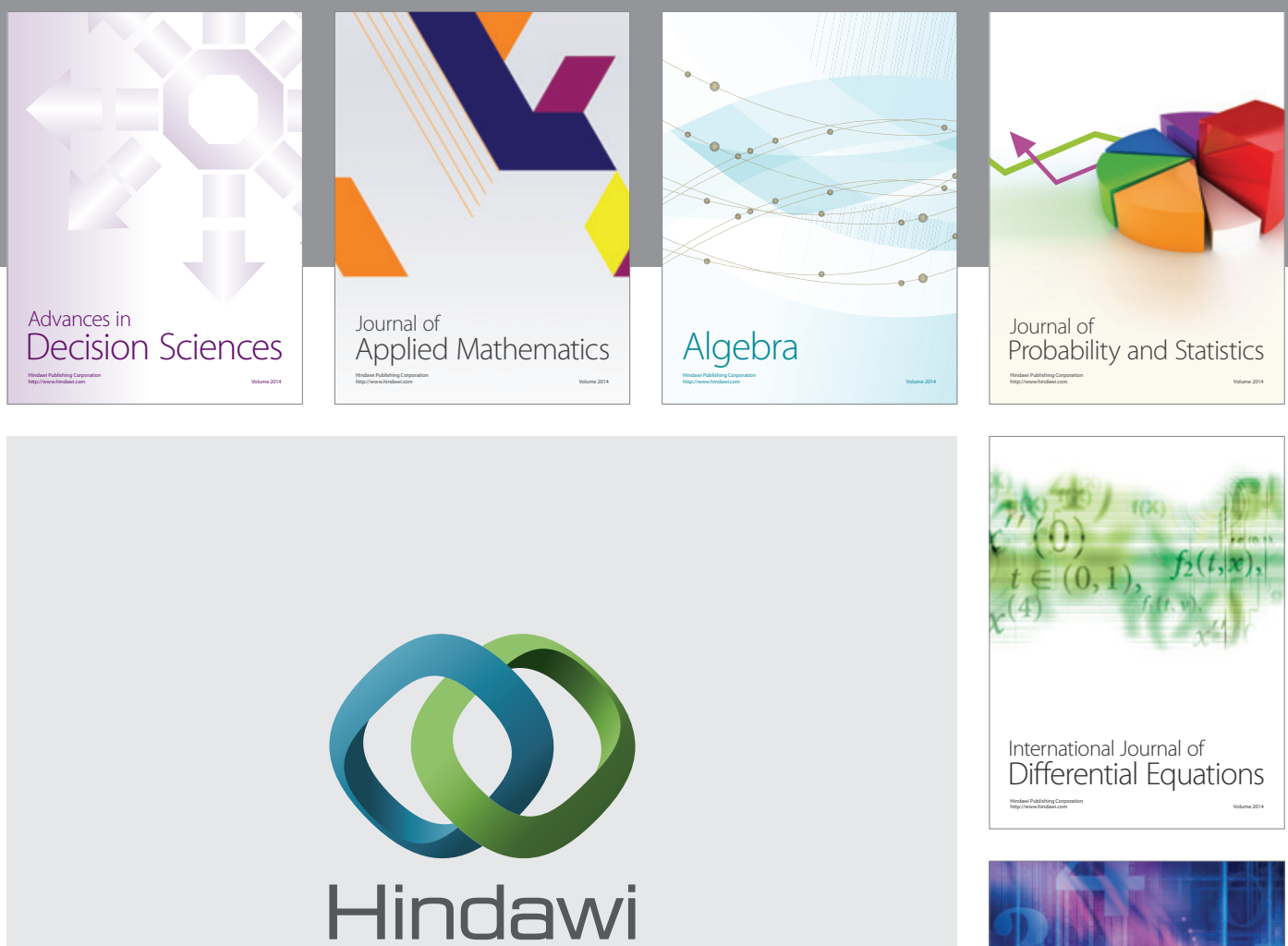

Submit your manuscripts at http://www.hindawi.com
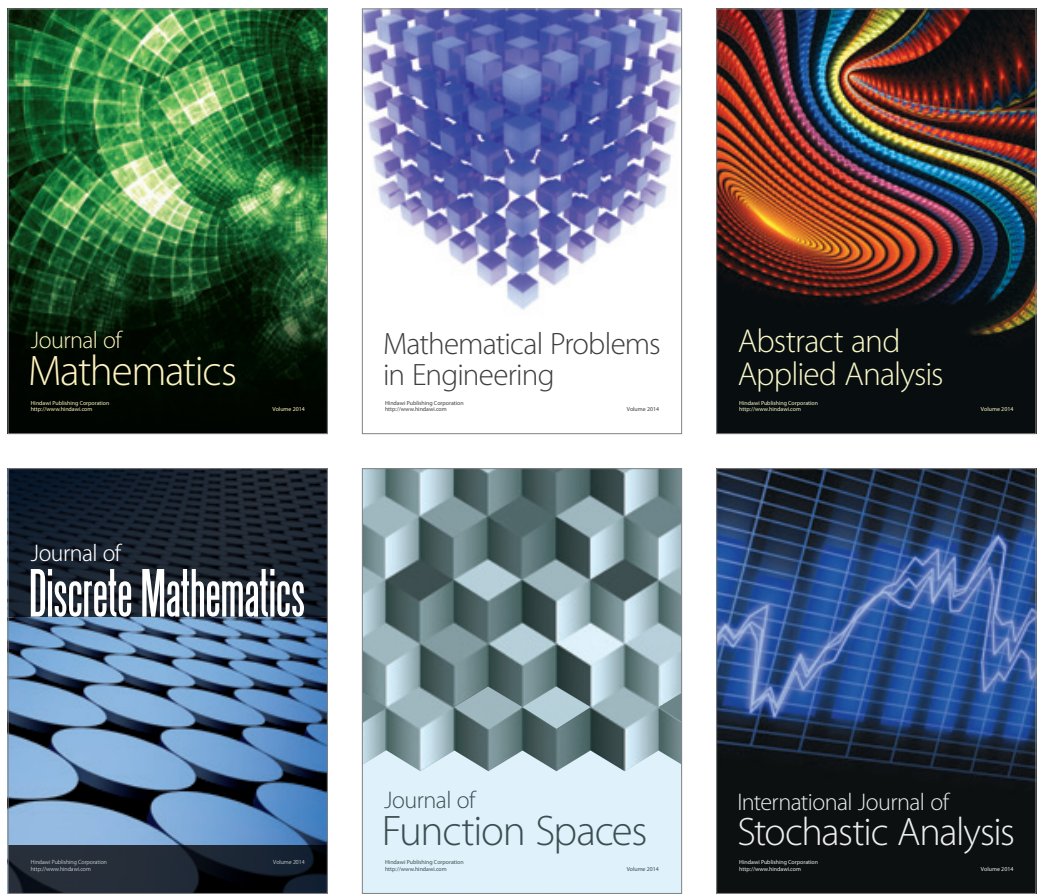

Journal of

Function Spaces

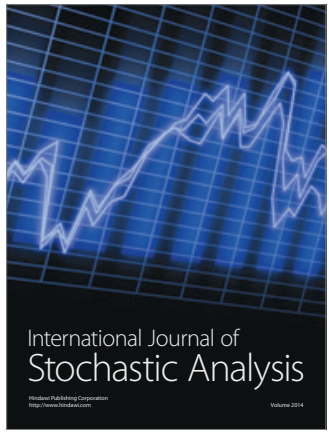

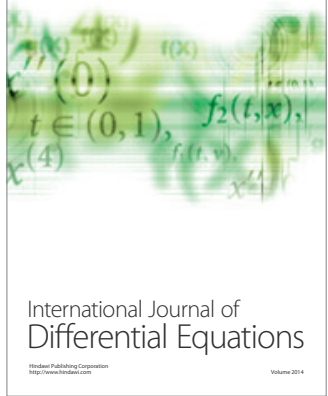
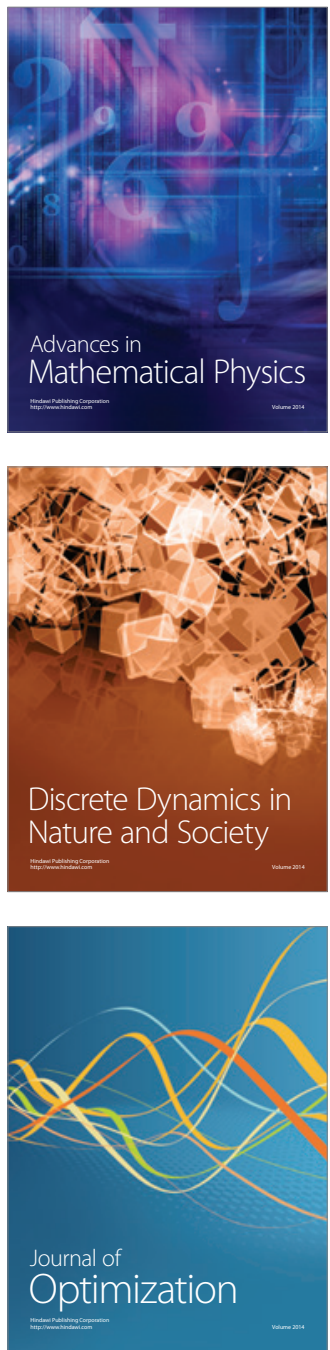